\title{
APLICAÇÃO DA TÉCNICA DE ELETRODIAGNÓSTICO \\ EM PACIENTES CRÍTICOS: UMA REVISÃO SISTEMÁTICA
}

\author{
APPLICATION OF ELECTRODIAGNOSTIC TECHNIQUE \\ IN CRITICALLY ILL PATIENTS: A SYSTEMATIC REVIEW
}

\begin{abstract}
Amanda Sachetti ${ }^{1,2}$, Janaína Pilau², João Luiz Quagliotti Durigan³, Paulo Eugênio da Silva ${ }^{3}$, Alexandre Simões Dias ${ }^{4,5}$
\end{abstract}

\footnotetext{
Clin Biomed Res. 2019;39(3):231-250

1 Faculdade de Medicina, Imed. Passo Fundo, RS, Brasil.

2 Unidade de Terapia Intensiva, Hospital de Clínicas de Passo Fundo (HCPF). Passo Fundo, RS, Brasil.
}

3 Grupo de Pesquisa em Plasticidade
Musculotendínea, Universidade de
Brasília (UnB). Ceilândia, DF, Brasil.

4 Programa de Pós-graduação em Ciências Pneumológicas e Ciências do Movimento Humano, Universidade Federal do Rio Grande do Sul (UFRGS). Porto Alegre, RS, Brasil.

5 Serviço de Fisioterapia, Hospital de Clínicas de Porto Alegre (HCPA). Porto Alegre, RS, Brasil.

Autor correspondente: Amanda Sachetti amandasachetti@gmail.com Faculdade de Medicina, Imed Rua Senador Pinheiro, 304. 99070-220, Passo Fundo, RS, Brasil.

\section{RESUMO}

O ambiente de terapia intensiva está evoluindo em tecnologias para avaliação e tratamento sendo uma das técnicas mais atuais aplicadas neste ambiente o eletrodiagnóstico. Esta é uma revisão sistemática desenvolvida com base nos resultados de pesquisa das principais bases de dados, seguindo o PRISMA. Neste foram incluídos somente artigos observacionais que utilizaram a técnica de eletrodiagnóstico em pacientes críticos, para os mais diferentes objetivos e desfechos. As bases de dados consultadas foram: MEDLINE (acessado via PubMed), Fisioterapia Evidence banco de dados (Pedro), Registro de Ensaios Controlados (CENTRAL Cochrane) e EMBASE além de uma busca manual de referências adicionais. Um total de 10 artigos foram encontrados, sendo que dois apresentaram-se repetidos e outros seis foram excluídos por não contemplarem os critérios de inclusão obtendo-se ao final um total de dois artigos totalizando 33 pacientes. Um dos artigos apresentou resultados seguros, sem lesão muscular e o protocolo foi viável para ser aplicado em terapia intensiva. O outro artigo que após lesão cerebral traumática os pacientes podem apresentar distúrbios eletrofisiológicos, além de atrofia muscular generalizada sendo evidenciados pela técnica de eletrodiagnóstico.

Palavras-chave: Unidade de terapia intensiva; ventilação mecânica; eletrodiagnóstico; cronaxia; músculo esquelético; respiração artificial

\section{ABSTRACT}

The intensive care environment is evolving in technologies for evaluation and treatment, and these include a recent technique named electrodiagnosis. This systematic review was based on search results from major databases, following PRISMA guidelines. Only observational studies using the electrodiagnostic technique in critically patients for different objectives and outcomes were included. The following databases were searched: MEDLINE (accessed via PubMed), Physiotherapy Evidence database (Pedro), Controlled Trials Registry (CENTRAL Cochrane) and EMBASE, in addition to a manual search for additional references. Ten articles were found, two of which were repeated and six were excluded because they did not meet the inclusion criteria; thus, two articles were selected, with a total of 33 patients. One of the articles showed safe results, without any muscle injury, and the protocol was applicable in intensive care. The other article demonstrated that, after traumatic brain injury, patients may present with electrophysiological disorders and generalized muscle atrophy, which can be revealed by the electrodiagnostic technique.

Keywords: Intensive care unit; mechanical ventilation; electrodiagnosis; chronaxia; skeletal muscle; artificial respiration 
Os cuidados em terapia intensiva vêm se desenvolvendo ao longo do tempo e por meio da tecnologia, prolongando a vida e melhorado as condições de saúde dos pacientes críticos. No entanto, o aumento no tempo de internação acompanha a perda de força muscular geralmente associada a atrofia, sendo clinicamente evidente em cerca de $25 \%$ dos pacientes após vários dias de ventilação mecânica ${ }^{1-3}$. Diante dessas questões, os profissionais de saúde foram desafiados a avaliar, mensurar e melhorar o status funcional em pacientes criticamente doentes, estando dentro deste grupo de profissionais os fisioterapeutas ${ }^{4-6}$.

Uma das características negativas do tempo de permanência prolongado na UTI é a fraqueza muscular, caracterizada por uma diminuição da excitabilidade neuromuscular, generalizada paresia e dificuldade de desmame devido à $\mathrm{VMI}^{7}$. Portanto, observou-se por parte dos profissionais, a necessidade de avaliar as alterações neuromusculares apresentadas, sendo o exame de eletrodiagnóstico uma possibilidade, por ser de baixo custo e não invasivo. Este teste de estímulo permite a aquisição de motor limiares de excitabilidade (reóbase e cronaxia) ${ }^{8,9}$. Dentro dos parâmetros determinados através do exame de eletrodiagnóstico, os principais são: a construção das curvas de intensidade-duração do pulso (através de correntes de pulso retangulares e exponenciais), a determinação da reóbase, que é a intensidade mínima da corrente para promover uma contração muscular com tempo de pulso muito largo (que em termos fisiológicos é 1 segundo), a determinação da cronaxia, que é o tempo de pulso para promover uma contração muscular usando-se uma intensidade duas vezes o valor da reóbase, a determinação da capacidade que os músculos Têm de se acomodarem a pulsos largos de subida lenta (acomodação) ${ }^{10,11}$.

Como parâmetros, considera-se que em músculos com a inervação íntegra, os valores de reóbase e de cronaxia geralmente sejam baixos ${ }^{10-12}$. Já a lesão do nervo periférico tende a desenvolver uma hipoexcitabilidade ao longo do tempo ocorrendo então um aumento desses parâmetros de estimulação ${ }^{12-14}$.

Para tanto, considerando as diversas aplicações do exame de eletrodiagnóstico, como avaliação e acompanhamento do nível de excitabilidade dos músculos periféricos, bem como, para prescrição adequada de eletroestimulação neuromuscular. Este estudo tem como objetivo realizar uma revisão sistemática da literatura sobre como este exame vem sendo utilizado em UTIs.

\section{METODOLOGIA}

Trata-se de uma revisão sistemática desenvolvida com base no Preferred Reporting Items for Systematic Review and Meta-analyses: The PRISMA Statement ${ }^{14}$ e registrada no International prospective register of systematic reviews (Prospero) através do número CRD42018087361 em 11/02/2018.

\section{Critérios de Elegibilidade}

Foram incluídos estudos com metodologia observacional com pacientes internados em UTIs, que foram submetidos ao exame de eletrodiagnóstico, para os mais diferentes objetivos e desfechos. A escolha por estudos observacionais deu-se devido a inexistência de ensaios clínicos randomizados com essa temática, tanto na literatura nacional quanto internacional.

O desfecho incluído foi a aplicação do exame de eletrodiagnóstico em pacientes internados em UTIs.

Foram adotados os seguintes critérios de exclusão: estudos com metodologia não observacional, estudos com dados incompletos, resumos, resumos expandidos ou capítulos de livros.

\section{Estratégia de Busca}

Foram pesquisados os seguintes bancos de dados eletrônicos: MEDLINE (acessado via PubMed), Physiotherapy Evidence Database (PEDro), Register of Controlled Trials (Cochrane CENTRAL) e EMBASE. Ainda, foi realizada uma busca complementar manual nas referências de estudos já publicados sobre o assunto. A busca foi realizada em janeiro de 2017 e compreendeu os seguintes descritores e seus termos sinônimos: "critical illness", "intensive care", "intensive care units" e "chronaxie", associados a uma sensível lista de termos para busca de estudos observacionais. Não houve restrição de idioma nem de datas limites para a busca. A estratégia de busca completa utilizada para o PubMed pode ser observada na Tabela 1.

Após a busca completa, todos os títulos e resumos foram avaliados por dois revisores de forma independente. Aqueles artigos que não forneceram em seu resumo as informações suficientes foram selecionadas para avaliação dos textos completos. Após a seleção por título e resumo, os mesmos revisores avaliaram independentemente os artigos completos e fizeram suas seleções, sendo que as discordâncias entre os revisores foram sanadas por consenso.

A extração dos dados foi realizada através de um formulário padronizado pelos mesmos dois revisores em duplicata. $O$ desfecho principal extraído foi: aplicação do exame de eletrodiagnóstico em pacientes em VMI internados em UTIs. 
Tabela 1: Estratégia de busca utilizada no PubMed.

\#1 (“Critical IIIness"[Mesh] OR “Critical IIIness" OR "Critical Illnesses" OR "Illness, Critical” OR "IIInesses, Critical" OR "Critically III" OR "Intensive Care"[Mesh] OR "Intensive Care" OR "Care, Intensive" OR "Surgical Intensive Care" OR "Care, Surgical Intensive" OR "Intensive Care, Surgical" OR "Intensive Care Units"[Mesh] OR "Intensive Care Units" OR "Care Unit, Intensive" OR "Care Units, Intensive" OR "Intensive Care Unit" OR "Unit, Intensive Care" OR "Units, Intensive Care" OR "critical illness polyneuromyopathy" OR "Polyneuropathy, Critical IIIness" OR "Critical IIIness Polyneuropathies" OR "Critical IIIness Polyneuropathy" OR "Polyneuropathies, Critical IIIness")

(Epidemiologic studies/) OR (Exp case control studies/) OR (Exp cohort studies/) OR (Case control.tw.) OR

\#2 ((cohort adj (study or studies)).tw.) OR (Cohort analy\$.tw.) OR ((Follow up adj (study or studies)).tw.) OR ((observational adj (study or studies)).tw.) OR (Longitudinal.tw.) OR (Retrospective.tw.) OR (Crosssectional.tw.) OR (Cross-sectional studies/)

\#3 (“chronaxie"[mesh] OR "chronaxy")

\#4 (\#1 AND \#2 AND \#3)

Seleção dos estudos e extração dos dados.

\section{Avaliação do Risco de Viés}

A avaliação da qualidade metodológica foi realizada descritivamente por dois revisores, de acordo com a escala The Newcastle-Ottawa Scale (NOS) for Assessing the Quality of Nonrandomized Studies in Metanalysis ${ }^{15}$, a qual classifica os estudos em três pontos distintos sendo eles: seleção, comparabilidade e resultados, pontuados com estrelas em quantidade máxima de 3 para o item de seleção, 2 para comparabilidade e, no máximo 4 estrelas para 0 item resultados ${ }^{15}$.

\section{Análise de Dados}

A análise dos dados foi realizada de forma descritiva e qualitativa e está apresentada em forma de figuras e tabelas.

\section{RESULTADOS}

Foram encontrados na literatura através dos descritores escolhidos, um total de 10 artigos, sendo que 2 apresentaram-se repetidos e outros 6 foram excluídos por não contemplarem o desfecho determinado ${ }^{16-19}$. Os revisores, de forma independente, classificaram dois como aptos, totalizando 33 pacientes ${ }^{20,21}$ (Figura 1).

As características dos artigos selecionados quanto à intervenção e aos desfechos e resultados são apresentados na Tabela 2. Os dois estudos selecionados possuem alguns autores em comum, porém a amostra e os desfechos não têm relação entre si. Para tanto, apesar da aplicabilidade ser diferente, os parâmetros utilizados no eletrodiagnóstico são semelhantes, facilitando assim a comparação dos resultados obtidos.

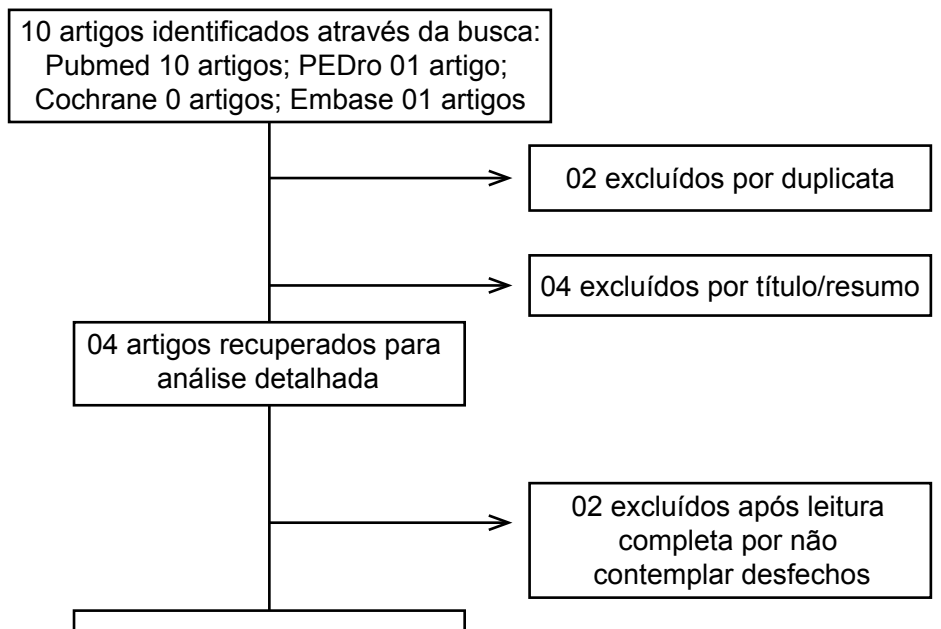

02 artigos incluídos

Figura 1: Fluxograma de seleção dos estudos para o desfecho definido pelos autores. 


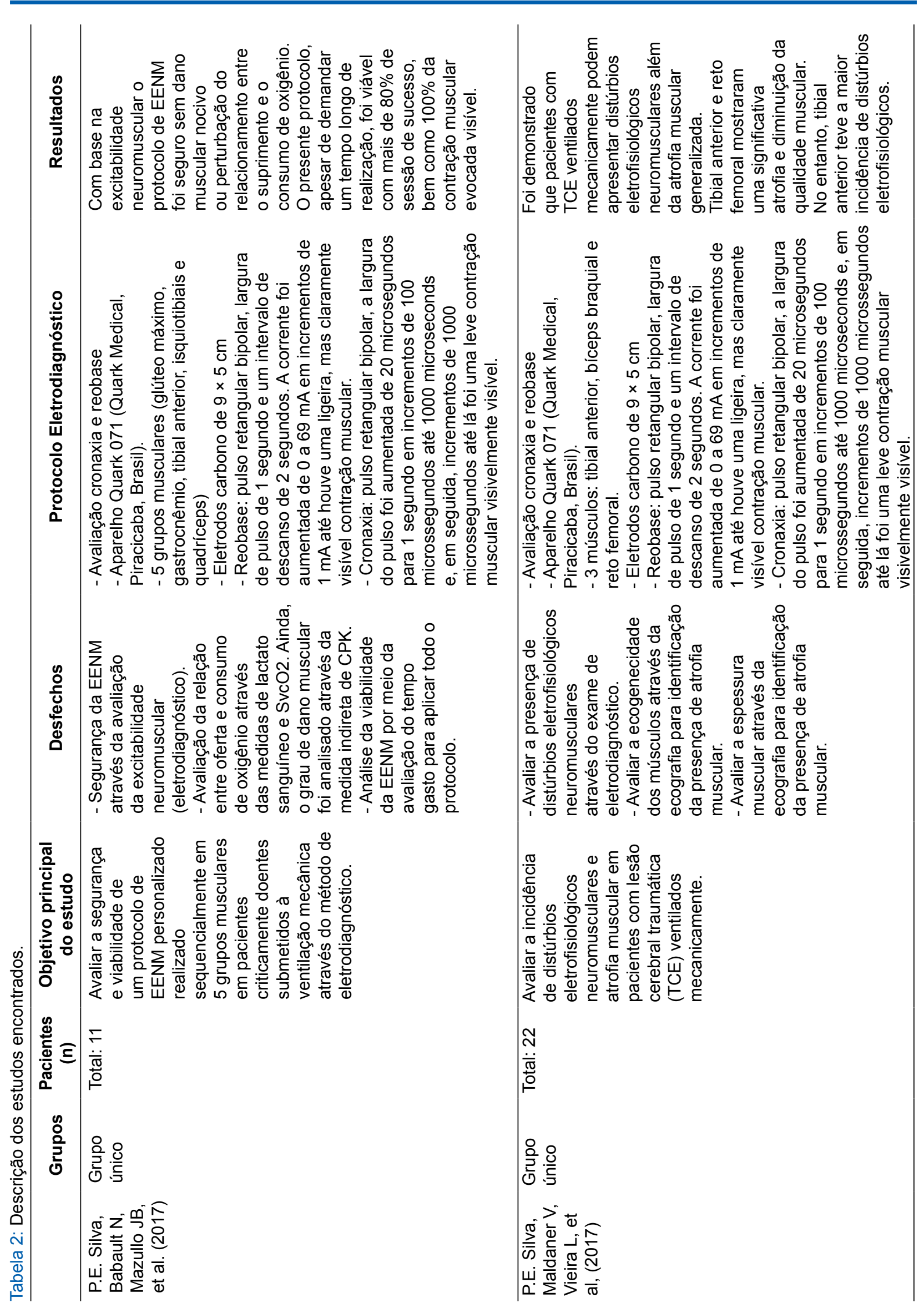


Ao comparar os artigos selecionados observa-se que apesar de terem sido desenvolvidos em UTIs, as características das amostras são diferentes (pacientes em VMI avaliados na admissão e seguidos por 3 dias versus pacientes com lesão cerebral traumática mas também em VMI e avaliados por 14 dias consecutivos), porém o desenho do estudo escolhido por ambos foi idêntico. Com relação ao tamanho das amostras foram 11 e 49 pacientes respectivamente ${ }^{20,21}$, portanto, amostras relativamente pequenas, possivelmente devido a complexidade dos critérios de inclusão e exclusão escolhidos.

O protocolo de eletrodiagnóstico utilizado foi semelhante entre os estudos, no entanto, os grupos musculares avaliados foram divergentes. Em uma delas os autores escolheram 5 grupos (tibial anterior, glúteo máximo, gastrocnêmio, reto femoral e isquiotibiais $)^{20}$ enquanto na outra foram 3 grupos musculares: bíceps braquial, reto femoral e tibial anterior ${ }^{21}$ entendendo que em ambos a escolha deu-se pela atuação na funcionalidade dos indivíduos.
Quanto aos resultados, um dos manuscritos não apresentou dados de alteração de excitabilidade neuromuscular ${ }^{20}$ enquanto o outro mostrou ao longo da internação quase $50 \%$ de pacientes portadores de disfunção ${ }^{21}$, o que pode ser explicado pelas características das amostras e ainda, pela diferença entre os momentos em que as análises foram realizadas.

Em relação ao risco de viés, classificado pela escala NOS, os artigos apresentaram resultados diferentes no item de seleção, onde o máximo de estrelas possíveis eram 4, considerando que um deles apresentou 3 estrelas ${ }^{20}$ e o outro ${ }^{21}$ apresentou 2 estrelas. Para o item de comparabilidade ambos os estudos foram classificados com 1 estrela, de um máximo de 2. Já para o item exposição, ambos apresentaram 3 estrelas, sendo este o máximo possível. A classificação se dá por meio da avaliação da quantidade de estrelas, que quanto maior, menor o risco de viés (Tabela 3 ).

Tabela 3: Risco de viés.

\begin{tabular}{llll}
\hline Estudo & Seleção & Comparabilidade & Resultado \\
\hline Silva, PE; Maldaner V; Vieira L, et al; 2017 & &
\end{tabular}

Risco de viés segundo a classificação de Newcastle Otawa (NOS) para estudos de coorte.

\section{DISCUSSÃO}

Considerando o grau de fraqueza muscular adquirida por pacientes em $\mathrm{VMI}$, os quais permanecem por períodos prolongados de tempo no leito, a importância da análise eletrofisiológica das estruturas musculares destes pacientes está bem estabelecida na literatura ${ }^{7,22}$, e o exame padrão ouro para estes casos é a eletroneuromiografia com eletrodo de agulha, embora não seja hoje uma rotina nas UTIs ${ }^{23}$ devido a ausência de equipamentos, ou pela falta de profissionais especializados na realização e interpretação deste exame, além de ser um procedimento invasivo. Para tanto, os estudos incluídos nesta pesquisa abordam o uso do eletrodiagnóstico para facilitar a avaliação desse grupo de pacientes como uma proposta novo ainda pouco estudada.

Após uma vasta busca na literatura, apenas 10 artigos foram encontrados utilizando uma lista sensível de termos de busca citados na metodologia, sendo que destes, somente 2 atingiam os critérios de inclusão, repetindo alguns autores em ambos, o que nos leva a crer que este tema apesar de sua necessidade emergente ainda é pouco explorado pelos profissionais fisioterapeutas intensivistas.

Os artigos encontrados trazem enfoques diferentes: um deles ${ }^{21}$ tem como amostra os pacientes com trauma crânio encefálico (TCE) e a aplicação do eletrodiagnóstico para avaliação da presença de atrofia muscular e distúrbios eletrofisiológicos, considerando que esta classe de pacientes passa por um prolongado tempo de imobilidade sem ser possível avaliar a perda de força muscular de forma ativa. Este teste não específico não permite que o examinador determine a fisiopatologia da lesão neurogênica ou mesmo a contribuição da miopatia nos distúrbios eletrofisiológicos. No entanto, ele pode ser usado como um exame inicial de triagem e seus resultados podem fornecer uma linha de ação racional para o estudo da condução nervosa e estimulação muscular direta subsequentes em configurações de UTIs ${ }^{24}$.

Neste estudo ${ }^{21}, 3$ grupos musculares foram avaliados, onde o tibial anterior e o reto femoral mostraram uma significativa atrofia e diminuição da qualidade muscular, mostrando altos valores de cronaxia. No entanto, tibial anterior foi o músculo que apresentou maior incidência de distúrbios eletrofisiológicos, apesar de ser pequeno, contudo, os autores afirmam que deve ser sempre considerado para o tratamento e/ ou monitoramento por ter um papel importante para a independência e a velocidade de caminhada em pacientes com deficiência ${ }^{25,26}$. É possível sugerir que o tratamento deste músculo pode fornecer 
maior funcionalidade para os sobreviventes da UTI. Essa incidência predominante no tibial anterior reforça as publicações anteriores utilizando testes de biópsia e / ou angiografia eletromiográfica que demonstraram maior incidência de axonopatia no nervo peroneal ${ }^{22,27,28}$. Alguns estudos nacionais e internacionais não realizados em UTIs utilizaram vários grupos musculares de forma concomitante para uma avaliação mais da excitabilidade, sendo esta uma opção indicada para ser realizada em novos protocolos com a população crítica ${ }^{21,22,29}$.

Já no outro estudo selecionado nesta revisão ${ }^{20}$, os autores buscaram avaliar a segurança e viabilidade de um protocolo de EENM personalizado realizado sequencialmente em 5 grupos musculares em pacientes criticamente doentes submetidos à VMI. Os mesmos tinham como hipótese que o protocolo de EENM proposto seria seguro, não promovendo distúrbios na relação entre oferta e consumo de oxigênio e danos musculares analisados por meio do exame de eletrodiagnóstico. Considerando o desfecho principal dos autores, o uso da técnica em questão teve como objetivo a padronização de um protocolo terapêutico, considerando-a um guia para a intervenção realizada. Ainda, os autores encontraram valores diferentes de cronaxia e reobase para os grupos musculares estudados indicando que o uso do eletrodiagnóstico como um norteador para a prática clínica têm relevância.

O estudo de Peviani e colaboradores ${ }^{30}$ mostrou que EENM com base em cronaxia e reobase é segura e eficaz na promoção do anabolismo da expressão gênica em camundongos com deficiência da excitabilidade neuromuscular. Recentemente, isso foi confirmado em humanos com o músculo tibial anterior usando um protocolo de EENM personalizado baseado em valores de cronaxia ${ }^{9}$. O teste de eletrodiagnóstico de estímulo permite para a aquisição da cronaxia que tem um papel fundamental no tratamento com EENM para pacientes com distúrbios eletrofisiológicos neuromusculares ${ }^{9}$.

Dos estudos incluídos nesta revisão, um deles ${ }^{20}$ não encontrou alterações de excitabilidade, já o outro ${ }^{21}$, que analisou os mesmos parâmetros por 14 dias, próximo ao período final da análise, observou uma incidência de $48 \%$ de lesão. Apesar de ambos terem avaliado o mesmo musculo, tibial anterior, as diferenças nos resultados devem dar-se, provavelmente, pelo período em que a avaliação foi feita: no primeiro ${ }^{20}$ ocorreu logo após a internação na UTI e seguiu por apenas três dias consecutivos, enquanto o outro ${ }^{21}$ teve como seguimento 14 dias, observando maior taxa de alteração excitável entre 7 e 14 dias. Estes resultados inferem que os maiores níveis de comprometimento ocorrem com o prolongamento da estadia do paciente na UTI não sendo confiável a avaliação por eletrodiagnóstico em somente um momento, mas que é necessário um acompanhamento da evolução do paciente ao longo de sua internação.

\section{CONCLUSÃO}

O exame de eletrodiagnóstico vem sendo amplamente estudado, porém ainda de forma discreta em UTIs, apesar de os resultados encontrados até o presente momento se mostrarem positivos para diversas aplicabilidades. Considera-se que os resultados encontrados nos dois artigos desta revisão sirvam como fator encorajador para novas pesquisas em âmbito de terapia intensiva testando as funções do exame de eletrodiagnóstico.

\section{REFERÊNCIAS}

1. De Jonghe B, Sharshar T, Lefaucheur JP, Authier FJ, DurandZaleski I, Boussarsar M, et al. Paresis acquired in the intensive care unit: a prospective multicenter study. JAMA. 2002;288(22):2859-67.

2. Garnacho-Montero J, Madrazo-Osuna J, Garcia-Garmendia JL, Ortiz-Leyba C, Jiménez-Jiménez FJ, Barrero-Almodóvar A, et al. Critical illness polyneuropathy: risk factors and clinical consequences. A cohort study in septic patients. Intensive Care Med. 2001;27(8):1288-96.

3. De Letter MA, Schmitz PI, Visser LH, Verheul FA, Schellens RL, Op de Coul DA, Van der Meché FG. Risk factors for the development of polyneuropathy and myopathy in critically ill patients. Crit Care Med. 2001;29(12):2281-6.

4. Jones AYM. Evidence-based physiotherapy in intensive care. Hong Kong Physioth J. 2000;18(2):47-52.

5. Denehy L, Berney S. Physiotherapy in intensive care unit. Phys Ther Rev. 2006;11(1):49-56.

6. Stiller K. Physiotherapy in intensive care: towards an evidence-based practice. Chest. 2000;118(6):1801-13.

7. Lacomis, D. Electrophysiology of neuromuscular disorders in critical illness. Muscle Nerve. 2013;47(3):452-63.
8. Fernandes LFRM, Oliveira NML, Pelet DCS, Cunha AFS, Grecco MAS, Souza LAPS. Stimulus electrodiagnosis and motor and functional evaluations during ulnar nerve recovery. Braz J Phys Ther. 2016;20(2):126-32.

9. Pieber K, Herceg M, PaternostroSluga T, Schuhfried O. Optimizing stimulation parameters in functional electrical stimulation of denervated muscles: a cross-sectional study. $J$ Neuroeng Rehabil. 2015;7:12-51.

10. Polônio JT, Mazzer N, Barbieri CH, Mattielo-Sverzut AC. Eletroestimulação seletiva mantem estrutura e função do tibial anterior desenervado de ratos. Acta Ortop Bras. 2010;18(2):85-9. 
11. Russo TL, Franca CN, Castro CES, Salvini TF. Alterations of chronaxie, rheobase and accommodation in denervated skeletal muscle submitted to electrical stimulation. Braz J Phys Ther. 2004;8(2):169-75.

12. Ashley $Z$, Sutherland $H$, Lanmuller $H$, Unger E, Li F, Mayr W, et al. Determination of the chronaxie and rheobase of denervated limb muscles in conscious rabbits. Artif Organs. 2005;29(3):212-5.

13. Gutmann E, Zelena J. Morphological change in the denervated muscle. In: Gutmann E, editor. The denervated muscle. Prague: Czech Academy of Sciencies. 1962. p. 57-102.

14. Castro CES, Longo GJ, Parizotto NA. A importância do eletrodiagnóstico de estímulo na terapia excitomotora. Rev Paul Fisioter. 1980;2:54-68.

15. Wells GA, Shea B, O'Connell D, Peterson J, Welch V, Losos M Tugwell P. The Newcastle-Ottawa Scale (NOS) for assessing the quality of non-randomized studies in metaanalyses [Internet]. Ottawa: Ottawa Hospital Research Institute; 2001 [citado 2019 Out 16]. Disponível em: http://www.ohri.ca/programs/clinical_ epidemiology/oxford.asp

16. Gibbels E, Schliep G. Diabetic polyneuropathy: problems of diagnosis and nosology. Demonstrated on the basis of recent literature reviews and analysis of 100 cases. Fortschr Neurol Psychiatr Grenzgeb. 1970;38(8): 369-436.

17. Kimura W. Electrodiagnostic study of the facial nerve in Charcot-MarieTooth disease and the Guillain-Barré syndrome. Neurology. 1976:20:28.
18. Reniers J, Martin L. Denervation atrophy in isoniazid polyneuropathy. Histo-enzymatic study. Acta Neurol Psychiatr Belg. 1967;67(10):783-96.

19. Matiar-Vahar H, Rohrer H. Familial recurrent polytopic neuropathy. Fortschr Neurol Psychiatr Grenzgeb. 1970;38(10):493-523.

20. Silva PE, Babault N, Mazullo JB, Oliveira TP, Lemos BL, Carvalho VO, Durigan JLQ. Safety and feasibility of a neuromuscular electrical stimulation chronaxie-based protocol in critical ill patients: a prospective observational study. J Crit Care. 2017;37:1418.

21. Silva PE, Maldaner V, Vieira L, Carvalho KL, Gomes H, Melo P, et al. Neuromuscular electrophysiological disorders and muscle atrophy in mechanically-ventilated traumatic brain injury patients: new insights from a prospective observational study. $J$ Crit Care. 2017;19(44):87-94.

22. Latronico N, Bertolini G, Guarneri B, Botteri M, Peli E, Andreoletti S, et al. Simplified electrophysiological evaluation of peripheral nerves in critically ill patients: the Italian multicentre CRIMYNE study. Crit Care. 2007;11(1):1-11.

23. Parry SM, El-Ansary D, Cartwright MS, Sarwal A, Berney S, Koopman R, et al. Ultrasonography in the intensive care setting can be used to detect changes in the quality and quantity of muscle and is related to muscle strength and function. J Crit Care. 2015;30(5): 1151.e9-14.

24. Paternostro-Sluga T, Schuhfried O, Vacariu G, Lang T, Fialka-Moser V. Chronaxie and accommodation index in the diagnosis of muscle denervation. Am J Phys Med Rehabil. 2002;81(4):253-60.
25. Itoh S, Kubota K, Ogata K, Tsuji T. Exercise system for eccentric tibialis anterior contraction to improve ambulatory function. Conf Proc IEEE Eng Med Biol Soc. 2016;2016:5845-8.

26. Dorsch S, Ada L, Canning CG, Al-Zharani M, Dean C. The strength of the ankle dorsiflexors has a significant contribution to walking speed in people who can walk independently after stroke: an observational study. Arch Phys Med Rehabil. 2012;93(6):1072-6.

27. Latronico N, Fenzi F, Recupero D, Guarneri B, Tomelleri G, Tonin P, et al. Critical illness myopathy and neuropathy. Lancet. 1996;347(9015):1579-82.

28. Latronico N, Nattino G, Guarneri B, Fagoni N, Amantini A, Bertolini G, GiVITI Study Investigators. Validation of the peroneal nerve test to diagnose critical illness polyneuropathy and myopathy in the intensive care unit: the multicentre Italian CRIMYNE-2 diagnostic accuracy study. F1000Res. 2014;3:127.

29. Lee WD, Kim JH, Lee JU, Kim MY, Lee LK, Yang SM, et al. Differences in rheobase and chronaxie between the paretic and non-paretic sides of hemiplegic stroke patients: a pilot study. J Phys Ther Sci. 2013;25(6):7179.

30. Peviani SM, Russo TL, Durigan JLQ, Vieira BS, Pinheiro CM, Galassi MS, Salvini TF. Stretching and electrical stimulation regulate the metalloproteinase- 2 in rat denervated skeletal muscle. Neurol Res. 2010;32(8):891-6. 